\title{
Obturator Device
}

National Cancer Institute

\section{Source}

National Cancer Institute. Obturator Device. NCI Thesaurus. Code C50086.

A plug-like device used to close an opening in a body structure or a removable plug used during the insertion of a cannula. 